\title{
MODELING OF FLASHING-INDUCED FLOW INSTABILITIES FOR A NATURAL CIRCULATION DRIVEN NOVEL MODULAR REACTOR
}

\author{
Shanbin Shi* ${ }^{\mathrm{a} *}$ Mamoru Ishii ${ }^{\mathrm{b}}$
}

\begin{abstract}
An analytical study based on frequency domain analysis is presented on the flashing-induced flow instability in a natural circulation test facility, which was designed to investigate the flow instability for a BWR-type novel modular reactor (NMR). To address the flashing phenomena at low pressure conditions, such as initial startup transients or accidents, the liquid enthalpy change in the P-T diagram due to reduced hydrostatic head in the riser or chimney was treated as an axially uniform heat flux. Based on the drift flux model, the system transfer function was obtained through small perturbations about the steady state in the frequency domain. The D-partition method was used to determine the neutral stability boundary in the dimensionless stability plane, which was constituted of the subcooling number and phase change number. From the frequency domain analysis, the flashing stability boundary and the density wave oscillations boundary could be predicted. Some parametric studies had been performed on the system pressure and the inlet flow resistance coefficients in the stability analysis. The results showed that the flashing stability boundary was more sensitive to the system pressure than the density wave oscillations. In addition, the theoretical stability boundaries were benchmarked against the experimental stability boundaries from quasi-steady state tests. Although the general stability boundary agreed well with the experiments, certain discrepancies still existed due to the assumptions of thermal equilibrium in current study. In the future, the thermal non-equilibrium conditions including subcooled boiling will be taken into account in the flashing induced stability analysis.
\end{abstract}


Keywords: Flashing instability; Frequency domain analysis; Natural circulation test facility; Stability map. 


\section{Introduction}

The flashing induced stability problem, simplified as flashing instability, in two-phase flow system is of great importance to the safe operation of naturally driven nuclear reactors and passively safe engineering safety system in nuclear industry. The designs of naturally driven nuclear reactors, ranging from conventional nuclear reactors to small modular reactors [1], feature a relative long chimney section above the reactor core to increase the driving force of natural circulation. In addition, the design of the reactor cavity cooling system for the generation IV reactors characterizes a long riser above the cooling panel [2]. The flashing instability could easily occur in the chimney section due to reduced hydrostatic head during the initial startup transients of the nuclear reactors, or in the riser section for the reactor cavity cooling system during accidental scenarios. The void fraction increases in the chimney section due to the flashing evaporation. The flow fluctuations caused by the flashing instability usually have large magnitude depending on the system design. Therefore, the safe operation or the system performance would be largely affected by the flashing instability. Although the flashing instability have been widely investigated experimentally and numerically in the last decade [3-9], the difficulties of predicting the flashing instability still exists due to its complexity. The demand to improve and develop the mechanistic model for the flashing instability becomes much urgent and necessary especially with the advancement of the next generation nuclear plants (NGNP).

A recent research has been performed to investigate the flow instability in a Novel Modular Reactor (NMR) design for low power and low pressure conditions. The NMR developed at Purdue University is a BWR-type small modular reactor design, which relies on natural circulation to provide driving force for both normal operations and accidental management [10-12]. In previous research, a natural circulation test facility was scaled and designed from the NMR by using the three-level scaling method. The experimental study of startup transients of the NMR showed that more than one flow instability mechanisms occurred in the natural circulation test facility during the normal initial startup procedures [13, 14]. From the experimental research, two startup procedures, i.e., very slow startup procedures and pressurized startup procedures, were proposed for the initial startup procedures for the NMR [15] to eliminate the flow instabilities. In addition, the experimental stability maps were obtained under low pressures by performing quasi-steady state tests [16]. Following the experimental research, the theoretical stability analysis in the 
frequency domain has been carried out to predict the observed flow instabilities occurred in the test facility [17].

In this paper, a new flashing mechanistic model related to the flashing number combining with the linear frequency domain analysis under thermal equilibrium conditions have been developed to predict the flow instability boundary for the NMR. Section 2 introduces the fundamental basis for the frequency domain analysis, including the one-dimensional drift flux model, flashing instability model, detailed derivations of the kinematics and dynamics of the fluid in the riser section. Section 3 presents the experimental stability boundaries from the quasi-steady state tests in a small-scaled natural circulation test facility. Section 4 presents the theoretical stability maps and discusses the uncertainties between the theoretical and experimental stability maps. Key conclusions are summarized in Section 5.

\section{Frequency domain analysis}

The basic method used in this research to study the flashing instability is called linear frequency domain stability analysis, which can be used to obtain stability boundaries for a flow system. This method is a conventional way to analyze other flow instabilities such as the density wave oscillations (DWO) [1] and Ledinegg instability (flow excursion) etc. The frequency domain analysis in this research was based on the drift-flux model and its constitutive equations [18] for a two-phase flow system. For example, through applying a small perturbation about the steady state in the inlet flow velocity, the system transfer functions for the perturbed pressure drop along the channel could be obtained by linearizing the original non-linear partial differential equations. The system characteristic equation was solved to obtain the flow stability boundaries between the stable regions and unstable regions in the frequency domain. Ishii [19] applied this method to investigate the density wave oscillations and performed parametric studies of the heat flux, inlet subcooling, inlet velocity, system pressure, inlet and outlet $\mathrm{K}$ factor, etc. on the density wave oscillations. Saha [20] developed the thermal non-equilibrium model for the two-phase flow system based on Ishii's work. Since the flashing instability normally occurs in the chimney or riser section which is located downstream of a heated section, the frequency domain analysis is applied to the flow system including both the heated section and the chimney or riser section in current research. 


\subsection{Formulation of the Problem}

In order to predict the flow instability observed in the experiments analytically, the mathematical model is developed following Ishii's formulation of the DWO using conventional four-equation drift flux model [19]. The drift flux model with the flashing model integrated aims to be capable of predicting the flashing instability and the DWO for the NMR. The system of interest consists of four components of the NMR as shown in Fig. 1:

A) Single-phase upstream unheated region

B) Single-phase heated region with uniform core heat flux, $q^{\prime \prime}$

C) Two-phase heated region with uniform core heat flux, $q^{\prime \prime}$

D) Two-phase unheated region with assumed uniform flashing flux, $q_{f l}^{\prime \prime}$

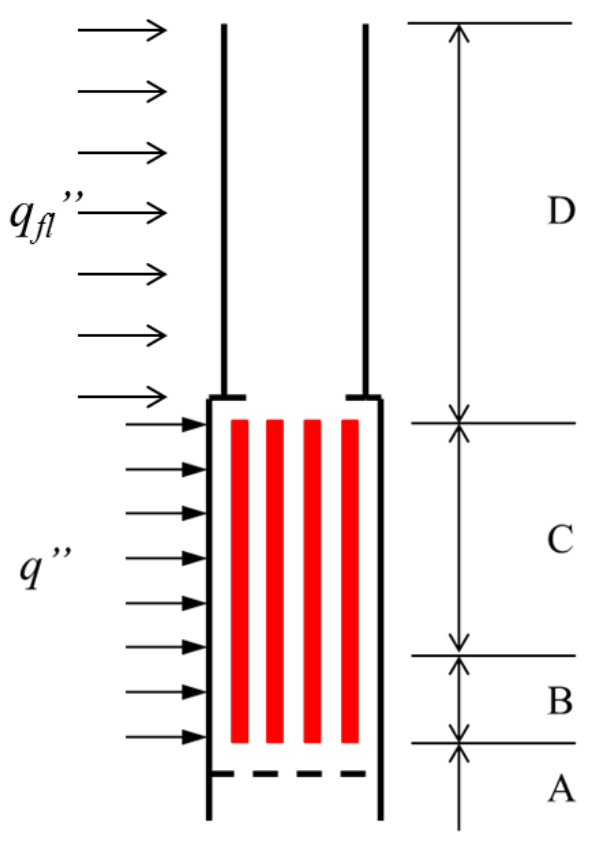

Fig. 1. System Used for Analysis of Flow Instability

\subsection{Field Equations}

The stability problems were formulated in terms of the time smoothed and area averaged field and constitutive equations. The area averaged one-dimensional drift flux model can be expressed as: 
Mixture continuity equation:

$$
\frac{\partial \rho_{m}}{\partial t}+\frac{\partial \rho_{m} v_{m}}{\partial z}=0
$$

Continuity equation for dispersed phase:

$$
\frac{\partial \alpha \rho_{g}}{\partial t}+\frac{\partial \alpha \rho_{g} v_{g}}{\partial z}=\Gamma_{g}
$$

Mixture momentum equation:

$$
\rho_{m}\left(\frac{\partial v_{m}}{\partial t}+v_{m} \frac{\partial v_{m}}{\partial z}\right)=-\frac{\partial p_{m}}{\partial z}-\frac{f_{m}}{2 D} \rho_{m} v_{m} v_{m}-\rho_{m} g-\frac{\partial}{\partial z}\left(\frac{\alpha_{g} \rho_{g} \rho_{f} V_{g j}^{2}}{\left(1-\alpha_{g}\right) \rho_{m}}\right)
$$

Mixture enthalpy-energy equation:

$$
\rho_{m}\left(\frac{\partial i_{m}}{\partial t}+v_{m} \frac{\partial i_{m}}{\partial z}\right)=\frac{q_{W}^{\prime \prime} \xi_{h}}{A}-\frac{\partial}{\partial z}\left(\frac{\alpha_{g} \rho_{g} \rho_{f} \Delta i_{f g} V_{g j}}{\rho_{m}}\right)
$$

where mixture properties are defined as

$$
\begin{gathered}
\rho_{m}=\alpha \rho_{g}+(1-\alpha) \rho_{f} \\
i_{m}=\frac{\alpha \rho_{g} i_{g}+\left(1-\alpha_{g}\right) \rho_{f} i_{f}}{\rho_{m}} \\
p_{m}=\alpha p_{g}+(1-\alpha) p_{f} \\
v_{m}=\frac{\alpha \rho_{g} v_{g}+(1-\alpha) \rho_{f} v_{f}}{\rho_{m}}
\end{gathered}
$$

and the drift velocity between two phases can be expressed as

$$
V_{g j}=(1-\alpha)\left(v_{g}-v_{f}\right)
$$

and the latent heat can be calculated as

$$
\Delta i_{f g}=i_{g}-i_{f}
$$

In the case of thermal and mechanical equilibrium conditions, the thermal properties of each phase are assumed saturated conditions. The kinematics and dynamics of the stability problem had been solved and obtained by Ishii [19] for the DWO without considering the flashing effects in the 
chimney section at high pressures. However, the flashing effect might be dominant at lower pressure transients for the natural circulation system, especially during the startup transients. With the flashing model in the chimney or riser section, the analysis method for the heated mixture Region $\mathrm{C}$ can be adopted to derive the kinematics and dynamics of the downstream un-heated Region D.

\subsection{Flashing Model}

In a natural circulation system, superheated liquid near the top of the riser or chimney can induce flashing due to the reduced hydrostatic head under low pressure conditions. It is very difficult to model the exact amount of the flashing vapor generation rate during such fast and thermal nonequilibrium transients. In current research, the vapor mass generation rate was simply derived based on the water P-T diagram as shown in Fig. 2 by assuming saturated conditions at the core exit. Thus, the liquid pressure changed from a higher pressure at the bottom of the chimney to an upward lower pressure. Correspondingly, the enthalpy change rate of the flashed liquid during this process could be calculated from the temperature change from the P-T diagram.

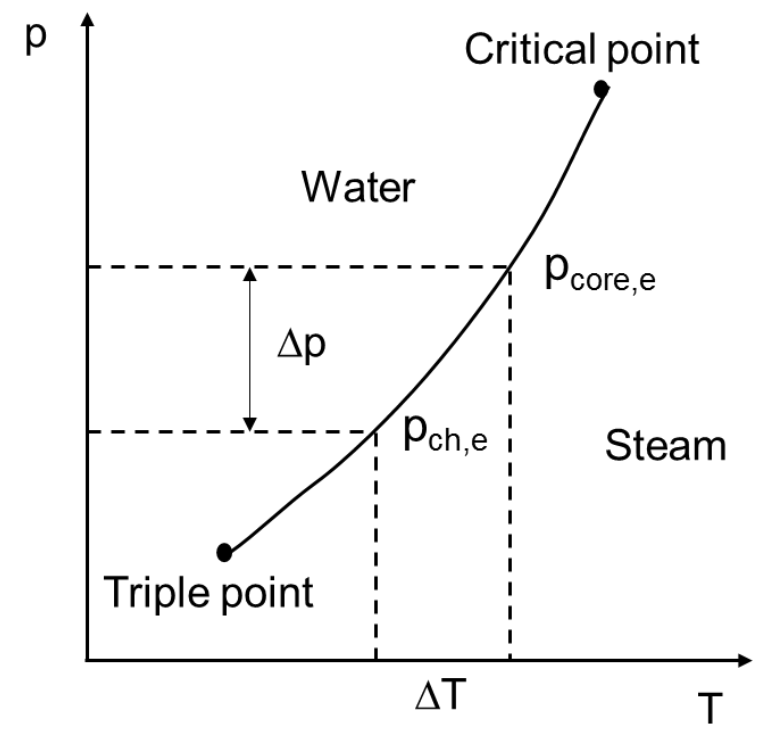

Fig.2. Flashing process in water P-T diagram 
Analogue to the core heating, the enthalpy change rate due to liquid flashing was assumed as a uniform heat source along the chimney. The assumed uniform flashing heat flux $q_{f l}^{\prime \prime}$ could be expressed as

$$
\begin{gathered}
q_{f l}^{\prime \prime}=j_{f} \rho_{f} c_{f} \Delta T \\
j_{f}=(1-\alpha) v_{f}
\end{gathered}
$$

Therefore, the flashing mass generation rate could be derived from the energy balance equation for a two-phase flow system as

$$
(1-\alpha) \rho_{f} v_{f} c_{f} \Delta T=\Gamma_{g, f l} \Delta i_{f g} l
$$

and thus

$$
\Gamma_{g, f l}=\frac{(1-\alpha) \rho_{f} v_{f} c_{f} \Delta T}{\Delta i_{f g} l}
$$

where, $\alpha, \rho_{f}, j_{f}, v_{f}, c_{f}, l, \Delta T, \Delta i_{f g}$, and $\Gamma_{g, f l}$ represented the void fraction at the core exit, liquid density, liquid volumetric flux, liquid velocity, specific heat, height of the riser or chimney, temperature difference corresponding to pressure differential based on P-T diagram, latent heat, and the vapor mass generation rate due to flashing, respectively. It was worthy of mention that the flashing process should include the core section if its elevation was so large that the reduced hydrostatic head could not be neglected. However, in this study, the flashing effect was neglected in the core section due to the relatively short core height in the NMR [9].

\subsection{Transfer Function and Stability Boundary}

In order to study the flashing instability, the total pressure drop perturbations of the system considered were analyzed with the introduction of an inlet velocity perturbation, $\delta v$. To obtain the stability boundary, the system transfer function, $1 / Q(s)$, was firstly obtained between the total pressure drop perturbations and the core inlet velocity perturbations as

$$
\delta v=\frac{1}{Q(s)} \delta \Delta p_{e x}
$$


where $Q(s)$ was system characteristic function in the system transfer function. According to control theory, the system asymptotic stability could be determined by the nature of roots of the characteristic equation given by

$$
Q(s)=0
$$

Normally, the characteristic equation was formulated in a series of dimensionless numbers as

$$
Q\left(s^{*}, \alpha_{1}, \alpha_{2}, \cdots, \alpha_{n}\right)=0
$$

where $\alpha_{1}$ to $\alpha_{n}$ were independent dimensionless numbers. For the reactor stability analysis, the phase change number and inlet subcooling number were selected to form the dimensionless stability plane ( $\mathrm{N}_{\mathrm{sub}}-\mathrm{N}_{\mathrm{pch}}$ ). If the harmonic oscillations were considered to simplify the solutions, system response could be obtained by substituting $s^{*}=j \omega^{*}$ into Eq. (17) as

$$
Q\left(j \omega^{*}, N_{p c h}, N_{s u b}\right)=Q_{\operatorname{Re}}\left(\omega^{*}, N_{p c h}, N_{s u b}\right)+j Q_{\operatorname{Im}}\left(\omega^{*}, N_{p c h}, N_{s u b}\right)=0
$$

Thus, Eq. (18) was reduced to

$$
\begin{aligned}
& Q_{\operatorname{Re}}\left(\omega^{*}, N_{p c h}, N_{s u b}\right)=0 \\
& Q_{\operatorname{Im}}\left(\omega^{*}, N_{p c h}, N_{s u b}\right)=0
\end{aligned}
$$

Equations (19) and (20) gave the harmonic frequency surfaces or neutral stability curves in the dimensionless plane of $\mathrm{N}_{\text {sub }}-\mathrm{N}_{\text {pch. }}$. The D-partition method [19, 21] stated that the number of roots lying in the right half $s^{*}$ plane for each region divided by surfaces do not change within a subdivision. Since the neutral stability curve was determined, the stability of each region could be determined by testing the stability at any point in that region using certain stability criteria such as Nyquist or Mikhailov Criterion used by Ishii [19]. The number of roots for the dimensionless characteristic Eq. (16) should be zero for the stability of the system.

\subsection{Time Lags and Space Lags}

Before the derivation of the transfer functions in the Region (D), two different methods, i.e. the Lagrangian and Eulerian specification of the flow field, to describe the enthalpy of the fluid particle were illustrated in Figs. 3 and 4, respectively. In Fig. 3, the particle entered region (A), (B), (C), 
and (D) at $\tau_{0}, \tau_{1}, \tau_{2}$, and $\tau_{3}$, respectively. The residence time between two time points was also called time lag, which was significantly related to the propagation of the density waves. Accordingly, the time lags were replaced by the space lags in Fig. 4, which defined the physical boundaries between two regions. One specially important space lag was the boundary between the single-phase liquid and two-phase mixture and was denoted by $\lambda$ corresponding to the time lag $\tau_{12} \cdot$

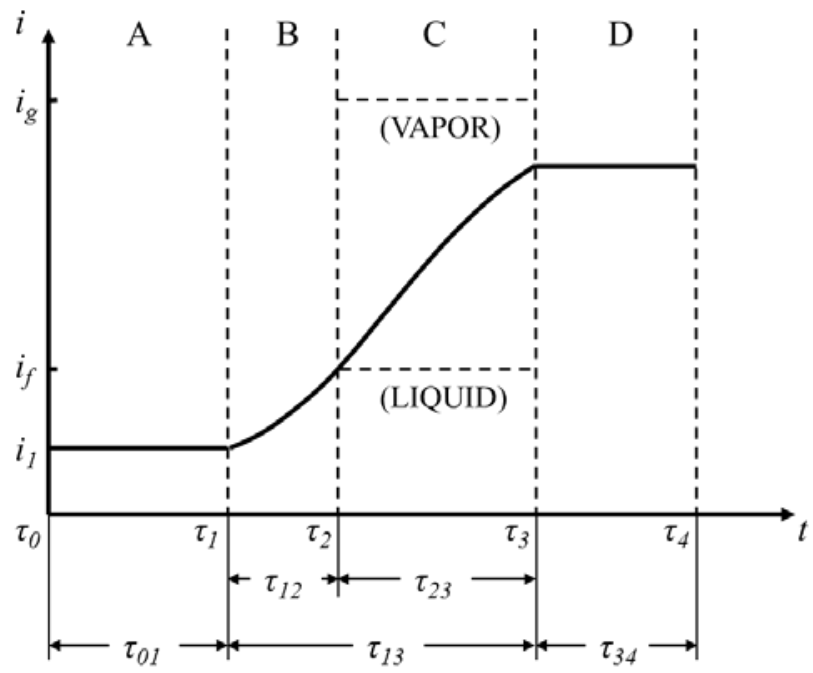

Fig. 3. Lagrangian description of enthalpy

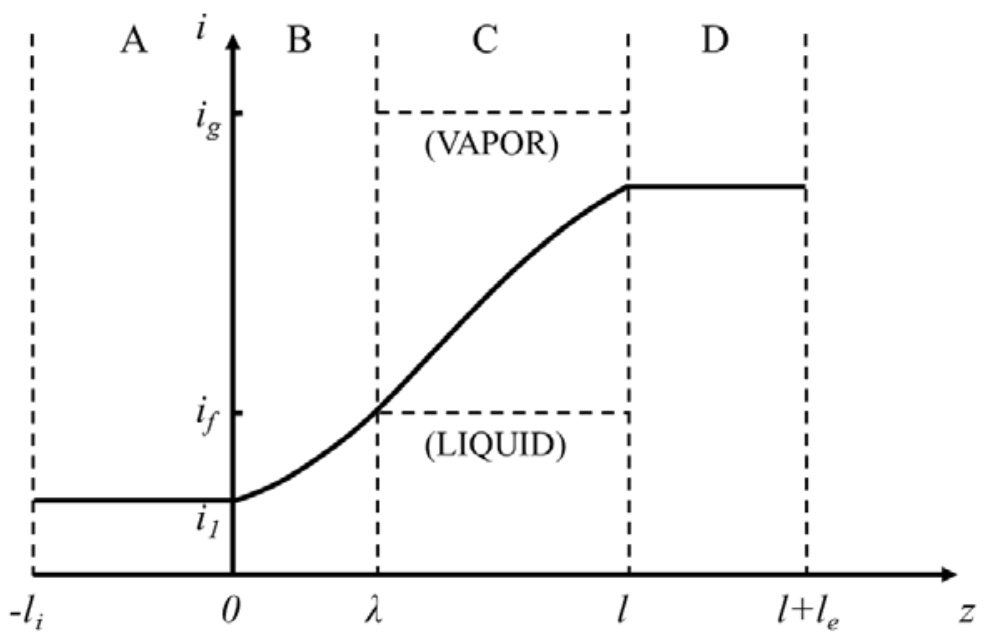

Fig. 4. Eulerian description of enthalpy 
2.6. Kinematics of the Un-heated Region D with Flashing

2.6.1. Volumetric flux equation and density propagation equation

The derivation of volumetric flux equation started from the continuity equations for each phase (vapor or liquid phase). Here the variables in this section are denoted by subscript $e$. Similar to the vapor phase in Eq.(2), the continuity equation for the liquid phase could be written as

$$
\frac{\partial(1-\alpha) \rho_{f}}{\partial t}+\frac{\partial(1-\alpha) \rho_{f} v_{f}}{\partial z}=\Gamma_{f}=-\Gamma_{g, f l}
$$

By carrying out the differentiations of the left hand side of Eqs. (2) and (21) as

$$
\frac{\partial \alpha}{\partial t}+\frac{\partial \alpha v_{g}}{\partial z}=\frac{\Gamma_{g, f l}}{\rho_{g}}-\frac{\alpha}{\rho_{g}} \frac{D_{g} \rho_{g}}{D t}
$$

and

$$
\frac{\partial(1-\alpha)}{\partial t}+\frac{\partial(1-\alpha) v_{f}}{\partial z}=-\frac{\Gamma_{f}}{\rho_{f}}-\frac{(1-\alpha)}{\rho_{f}} \frac{D_{f} \rho_{f}}{D t}
$$

By adding the above two equations and neglecting the compressibility effect, the volumetric flux equations could be obtained as

$$
\frac{\partial j_{e}}{\partial z}=\Gamma_{g, f l} \frac{\Delta \rho}{\rho_{g} \rho_{f}}
$$

where, the volumetric flux of the mixture was given by

$$
j_{e}=v_{m e}+\left(\frac{\rho_{f}}{\rho_{m e}}-1\right) V_{g j e}
$$

By considering the isochoric process for each phase, Eq. (22) could be expressed by $j_{e}$ and $V_{g j e}$ as

$$
\frac{\partial \alpha}{\partial t}+j_{e} \frac{\partial \alpha}{\partial z}+\frac{\partial\left(\alpha V_{g j e}\right)}{\partial z}=\frac{\rho_{m e} \Gamma_{g, f l}}{\rho_{f} \rho_{g}}
$$

Furthermore, by considering the vapor drift velocity $V_{g j}$ is a function of void fraction only, Eq. (26) could be re-written as

$$
\frac{\partial \alpha}{\partial t}+\left[j_{e}+V_{g j e}+\alpha \frac{\partial\left(\alpha V_{g j e}\right)}{\partial \alpha}\right] \frac{\partial \alpha}{\partial z}=\frac{\rho_{m e} \Gamma_{g, f l}}{\rho_{f} \rho_{g}}
$$


If we defined the kinematic wave velocity $C_{k e}$ by

$$
C_{k e}=j_{e}+V_{g j e}+\alpha \frac{\partial\left(\alpha V_{g j e}\right)}{\partial \alpha} \approx j_{e}+V_{g j e}
$$

Here, void propagation equation in this region could be expressed as

$$
\frac{\partial \alpha}{\partial t}+C_{k e} \frac{\partial \alpha}{\partial z}=\frac{\rho_{m e} \Gamma_{g, f l}}{\rho_{f} \rho_{g}}
$$

Under the condition of constant $\rho_{g}$ and $\rho_{f}$, the above equation could be expressed in terms of the mixture density as

$$
\frac{\partial \rho_{m e}}{\partial t}+C_{k e} \frac{\partial \rho_{m e}}{\partial z}=-\Gamma_{g, f l} \frac{\Delta \rho \rho_{m e}}{\rho_{g} \rho_{f}}
$$

where

$$
\Gamma_{g, f l}=\frac{c_{f}\left(T_{3}-T_{4}\right)(1-\bar{\alpha}) \bar{v}_{m, l} \rho_{f}}{l_{e} \Delta i_{f g}}
$$

$C_{k e}$, and $V_{g j e}$ was the kinematic wave velocity, drift velocity between two phases in this region, respectively. $\Gamma_{g, f l}$ was the vapor generation rate due to flashing in this region. $T_{3}$ and $T_{4}$ were the saturation temperatures corresponding to the pressures at the bottom and top of the chimney region, which were denoted by $p_{3}$ and $p_{4}$, respectively. $\bar{\alpha}$ and $\bar{v}_{m, l}$ were the steady state void fraction and mixture velocity at the exit of heated section, respectively. $l_{e}$ was the axial length of the downstream un-heated Region D. The characteristic frequency was expressed as

$$
\Omega_{e}=\Gamma_{g, f l} \frac{\Delta \rho}{\rho_{g} \rho_{f}}
$$

\subsubsection{Kinematic wave velocity}

The integration of Eq. (24) from the exit of the core to location $z$ in the chimney gave

$$
j_{e}(z, t)=j_{e}(l, t)+\Omega_{e}(z-l)
$$


By expanding $\rho_{m e}$ into $\bar{\rho}_{m e}+\delta \rho_{m e}(t)$ at the exit of the heated section, the equation for $C_{k e}$ became

$$
\begin{aligned}
& C_{k e}(z, t)=\bar{C}_{k e}(z)+\delta C_{k e}(t) \\
& =j_{e}(l, t)+V_{g j e}+\Omega_{e}(z-l) \\
& =v_{m e}(l, t)+\left[\frac{\rho_{f}}{\rho_{m e}(l, t)}-1\right] V_{g j e}+V_{g j e}+\Omega_{e}(z-l) \\
& =\bar{v}_{m e}(l)+\frac{\rho_{f}}{\bar{\rho}_{m e}(l)} V_{g j e}+\Omega_{e}(z-l)+\delta v_{m e}(l, t)-\frac{\rho_{f}}{\left[-\rho_{m e}(l)\right]^{2}} V_{g j e} \delta \rho_{m e}(l, t)
\end{aligned}
$$

where $V_{g j e}$ was assumed as a constant in Region D. The steady state part and the perturbation part of $C_{k e}$ could be written as

$$
\begin{gathered}
\bar{C}_{k e}(z)=\bar{v}_{m e}(l)+\frac{\rho_{f}}{\rho_{m e}(l)} V_{g j e}+\Omega_{e}(z-l) \\
\delta C_{k e}(t)=\delta v_{m e}(l, t)-\frac{\rho_{f}}{\left[\bar{\rho}_{m e}(l)\right]^{2}} V_{g j e} \delta \rho_{m e}(l, t)
\end{gathered}
$$

And the perturbation of mixture velocity and mixture density at the exit of the heated section was given in Ishii's thesis [19] as

$$
\begin{gathered}
\delta v_{m e}(l, t)=\varepsilon e^{s t}\left(\frac{A_{c}}{A_{e}}\right) \Lambda_{5}(l, s) \\
\delta \rho_{m e}(l, t)=\varepsilon e^{s t} \rho_{f} \Lambda_{6}(l, \mathrm{~s})
\end{gathered}
$$

So

$$
\delta C_{k, e}(t)=\varepsilon e^{s t} \Lambda_{20}(l, s)
$$

and the various transfer functions of $\Lambda_{n}(z, s)$ are given in Appendix A.

\subsubsection{Response of mixture density}


By now, the kinematic wave velocity was solved and could be substituted into Eq. (30) to obtain the density of the mixture. To simplify the derivation, a new variable was defined in this section as

$$
\phi_{e}(z, t)=\ln \left[\frac{\rho_{m e}(z, t)}{\bar{\rho}_{m e}(l)}\right]
$$

So the density propagation Eq. (30) became

$$
\frac{\partial \phi_{e}(z, t)}{\partial t}+C_{k e}(z, t) \frac{\partial \phi_{e}(z, t)}{\partial z}=-\Omega_{e}
$$

In order to apply perturbation method, we defined

$$
\phi_{e}(z, t)=\bar{\phi}_{e}(z)+\delta \phi_{e}(z, t)
$$

and

$$
\rho_{m e}(z, t)=\bar{\rho}_{m e}(z)+\delta \rho_{m e}(z, t)
$$

By using the order of magnitude analysis, $\bar{\phi}_{e}(z)$ and $\delta \phi_{e}(z, t)$ could be expressed as

$$
\begin{gathered}
\bar{\phi}_{e}(z)=\ln \left[\frac{\bar{\rho}_{m e}(z)}{\overline{\rho_{m e}(l)}}\right] \\
\delta \phi_{e}(z, t)=\frac{\delta \rho_{m e}(z, t)}{\bar{\rho}_{m e}(z)}
\end{gathered}
$$

Substituting Eqs. (42) and (34) into Eq. (41) and using the perturbation method, the zero ${ }^{\text {th }}$ order and first order equations were expressed as

$$
\bar{C}_{k e}(z) \frac{d \bar{\phi}_{e}(z)}{d z}=-\Omega_{e}
$$

and

$$
\frac{\partial \delta \phi_{e}(z, t)}{\partial t}+\bar{C}_{k e}(z) \frac{\partial \delta \phi_{e}(z, t)}{\partial z}=\delta \bar{C}_{k e}(t) \frac{\Omega_{e}}{\bar{C}_{k e}(z)}
$$

Integrating Eq. (46) from $l$ to $z$, then

$$
\bar{\phi}_{e}(z)-\bar{\phi}_{e}(l)=-\Omega_{e} \int_{l}^{z} \frac{1}{\bar{C}_{k e}(z)} d z=-\int_{l}^{z} \frac{d \bar{C}_{k e}(z)}{\bar{C}_{k e}(z)}=-\ln \left[\frac{\bar{C}_{k e}(z)}{\bar{C}_{k e}(l)}\right]
$$


Comparing Eqs. (44) and (48), the following equation could be obtained.

$$
\frac{\bar{\rho}_{m e}(z)}{\bar{\rho}_{m e}(l)}=\frac{\bar{C}_{k e}(l)}{\bar{C}_{k e}(z)}
$$

The solution of the perturbed part for the mixture density could be solved by transforming Eq. (47) to Lagrangian form as

$$
d t=\frac{d z}{\bar{C}_{k e}(z)}=\frac{\bar{C}_{k e}(z) d\left(\delta \phi_{e}\right)}{\delta C_{k e}(t) \Omega_{e}}
$$

If the particle entered the bottom of the chimney at $t=\tau_{3}$, the first equality in Eq. (50) could be integrated as

$$
t-\tau_{3}=\int_{l}^{z} \frac{d z}{\bar{C}_{k e}(z)}
$$

The right hand side of Eq. (51) was redefined as

$$
E_{e}(z)=\int \frac{d z}{\bar{C}_{k e}(z)}
$$

Thus Eq. (51) became

$$
t=\tau_{3}+E_{e}(z)-E_{e}(l)
$$

The second equality of Eq. (50) could be written as

$$
\begin{aligned}
& d\left(\delta \phi_{e}\right)=\frac{\delta C_{k e}(t)}{\left[\bar{C}_{k e}(z)\right]^{2}} d \bar{C}_{k e}(z)=\varepsilon e^{s t} \Lambda_{20}(l, s) \frac{1}{\left[\bar{C}_{k e}(z)\right]^{2}} d \bar{C}_{k e}(z) \\
& =\varepsilon e^{s \tau_{3}} e^{s\left[E_{e}(z)-E_{e}(l)\right]} \frac{\Lambda_{20}(l, s)}{\left[\bar{C}_{k e}(z)\right]^{2}} d \bar{C}_{k e}(z)
\end{aligned}
$$

By defining $H_{e}(z, s)$ as

$$
H_{e}(z, s)=\int e^{s\left[E_{e}(z)-E_{e}(l)\right]} \frac{1}{\left[\bar{C}_{k e}(z)\right]^{2}} d \bar{C}_{k e}(z)
$$

Integrating Eq. (54) from $l$ to $z$, the following equation could be obtained.

$$
\delta \phi_{e}\left(z, \tau_{3}\right)-\delta \phi_{e}(l)=\varepsilon e^{s \tau_{3}} \Lambda_{20}(l, s)\left[H_{e}(z, s)-H_{e}(l, s)\right]
$$


Thus $\phi_{e}\left(z, \tau_{3}\right)$ in the Region D was expressed as

$$
\begin{aligned}
& \phi_{e}\left(z, \tau_{3}\right)=\bar{\phi}_{e}(z)+\delta \phi_{e}\left(z, \tau_{3}\right) \\
& =\ln \left[\frac{\bar{C}_{k e}(l)}{\bar{C}_{k e}(z)}\right]+\left\{\delta \phi_{e}(l)+\varepsilon e^{s \tau_{3}} \Lambda_{20}(l, s)\left[H_{e}(z, s)-H_{e}(l, s)\right]\right\}
\end{aligned}
$$

And the boundary condition for the $\phi_{e}(z, t)$ was given as

$$
\phi_{e}\left(l, \tau_{3}\right) \approx 0
$$

Then

$$
\delta \phi_{e}(l)=0
$$

And $\delta \phi_{e}(z, t)$ in Eq. (57) could be written as

$$
\delta \phi_{e}(z, t)=\varepsilon e^{s t} e^{-s\left[E_{e}(z)-E_{e}(l)\right]} \Lambda_{20}(l, s)\left[H_{e}(z, s)-H_{e}(l, s)\right]
$$

Finally, the response of mixture density $\rho_{m e}(z, t)$ could be obtained from Eqs. (45), (49) and (60) as

$$
\begin{aligned}
& \frac{\rho_{m e}(z, t)}{\bar{\rho}_{m e}(l)}=\frac{\bar{\rho}_{m e}(z)}{\bar{\rho}_{m e}(l)}+\frac{\delta \rho_{m e}(z, t)}{\bar{\rho}_{m e}(l)} \\
& =\frac{\bar{C}_{k e}(l)}{\bar{C}_{k e}(z)}+\frac{\delta \rho_{m e}(z, t)}{\bar{\rho}_{m e}(z)} \frac{\bar{\rho}_{m e}(z)}{\bar{\rho}_{m e}(l)}=\frac{\bar{C}_{k e}(l)}{\bar{C}_{k e}(z)}+\frac{\bar{C}_{k e}(l)}{\bar{C}_{k e}(z)} \delta \phi_{e}(z, t)
\end{aligned}
$$

where

$$
\frac{\delta \rho_{m e}(z, t)}{\bar{\rho}_{m e}(l)}=\varepsilon e^{s t} \Lambda_{21}(z, s)
$$

\subsubsection{Center of mass velocity}

In this section, the solution of the mixture velocity was obtained after the solutions for the volumetric flux $j_{e}$ and mixture density $\rho_{m e}$ in the previous sections.

$$
v_{m e}=j_{e}-\left(\frac{\rho_{f}}{\rho_{m e}}-1\right) V_{g j e}
$$


Because $C_{k e}=j_{e}+V_{g j e}$, then

$$
\begin{aligned}
& v_{m e}(z, t)=C_{k e}(z, t)-V_{g j e}-\left[\frac{\rho_{f}}{\rho_{m e}(z, t)}-1\right] V_{g j e} \\
& =\bar{C}_{k e}(z)+\delta C_{k e}(t)-\frac{\rho_{f}}{\rho_{m e}(z, t)} V_{g j e} \\
& =\left[\bar{C}_{k e}(z)-\frac{\rho_{f}}{\bar{\rho}_{m e}} V_{g j e}\right]+\left[\delta C_{k e}(t)+\frac{\rho_{f}}{\rho_{m e}} \frac{\delta \rho_{m e}}{\rho_{m e}} V_{g j e}\right] \\
& =\left[\bar{C}_{k e}(z)-\frac{\rho_{f}}{\bar{\rho}_{m e}(l)} \frac{\bar{\rho}_{m e}(l)}{\bar{\rho}_{m e}} V_{g j e}\right]+\left[\delta C_{k e}(t)+\frac{\rho_{f}}{\bar{\rho}_{m e}(l)} \frac{\bar{\rho}_{m e}(l)}{\bar{\rho}_{m e}} \frac{\delta \rho_{m e}}{\bar{\rho}_{m e}} V_{g j e}\right] \\
& =\left[\bar{C}_{k e}(z)-\frac{\bar{C}_{k}(l) \bar{C}_{k e}(z)}{\bar{C}_{k}(\bar{\lambda}) \bar{C}_{k e}(l)} V_{g j e}\right]+\left[\delta C_{k e}(t)+\frac{\bar{C}_{k}(l) \bar{C}_{k e}(z)}{\bar{C}_{k}(\bar{\lambda}) \bar{C}_{k e}(l) \bar{\rho}_{m e}(l)} \frac{\delta \rho_{m e}}{\bar{\rho}_{m e}(l)} V_{g j e}\right] \\
& =\left[\bar{C}_{k e}(z)-\frac{\bar{C}_{k}(l) \bar{C}_{k e}(z)}{\bar{C}_{k}(\bar{\lambda}) \bar{C}_{k e}(l)} V_{g j e}\right]+\left[\delta C_{k e}(t)+\frac{\bar{C}_{k}(l) \bar{C}_{k e}(z)}{\bar{C}_{k}(\bar{\lambda}) \bar{C}_{k e}(l)} \frac{\delta \rho_{m e}}{\bar{\rho}_{m e}(l)} \frac{\bar{C}_{k e}(z)}{\bar{C}_{k e}(l)} V_{g j e}\right]
\end{aligned}
$$

By noticing $\bar{C}_{k}(l)=\bar{C}_{k e}(l)$ at the boundary between Regions C and D, Eq. (64) could be written

as

$$
\begin{aligned}
& \frac{v_{m e}(z, t)}{\bar{v}_{f i}}=\frac{\bar{C}_{k e}(z)}{\bar{C}_{k}(\bar{\lambda})}+\left[\frac{\delta C_{k e}(t)}{\bar{v}_{f i}}+\frac{1}{v_{f i}} \frac{\bar{C}_{k e}(z)}{\bar{C}_{k}(\bar{\lambda})} \frac{\bar{C}_{k e}(z)}{\bar{C}_{k e}(l)} \varepsilon e^{s t} \Lambda_{21}(z, s) V_{g j e}\right] \\
& =\frac{\bar{C}_{k e}(z)}{\bar{C}_{k}(\bar{\lambda})}+\frac{\varepsilon e^{s t}}{\bar{v}_{f i}} \Lambda_{22}(l, s)
\end{aligned}
$$

\subsubsection{Pressure drop of the downstream un-heated Region D with flashing}

The pressure drop response in the un-heated region could be obtained by integrating the momentum equation in this region.

$$
\begin{aligned}
& \Delta p_{34}=k_{e} \rho_{m e}(l) v_{m e}^{2}(l)+\int_{l}^{l+l_{e}}\left[\rho_{m e}\left(\frac{\partial v_{m e}}{\partial t}+v_{m e} \frac{\partial v_{m e}}{\partial z}\right)\right. \\
& \left.+g_{e} \rho_{m e}+\frac{f_{m e}}{2 D_{e}} \rho_{m e} v_{m e}^{2}+\frac{\partial}{\partial z}\left(\frac{\rho_{f}-\rho_{m e}}{\rho_{m e}-\rho_{g}} \frac{\rho_{f} \rho_{g}}{\rho_{m e}} V_{g j e}^{2}\right)\right] d z
\end{aligned}
$$


In real reactor, such as NMR, other terms could be neglected except the exit throttling $k_{e}$ and the gravitational term. The gravitational pressure drop was much bigger than other terms due to long chimney region design in natural circulation BWR.

$$
\begin{aligned}
& \Delta p_{34 g}=\int_{l}^{l+l_{e}} g_{e} \rho_{m e} d z=\int_{l}^{l+l_{e}} g_{e}\left(\bar{\rho}_{m e}+\delta \rho_{m e}\right) d z \\
& =g_{e} \int_{l}^{l+l l_{e}} \bar{\rho}_{m e}(l) \frac{\bar{C}_{k e}(l)}{\bar{C}_{k e}(z)} d z+g_{e} \int_{l}^{l+l_{e}} \delta \rho_{m e} d z \\
& =g_{e} \bar{\rho}_{m e}(l) \int_{l}^{l+l e} \frac{\bar{C}_{k e}(l)}{\bar{C}_{k e}(z)} d z+g_{e} \int_{l}^{l+l_{e}} \bar{\rho}_{m e}(l) \varepsilon e^{s t} \Lambda_{21}(z, s) d z \\
& =g_{e} \bar{\rho}_{m e}(l)\left[\int_{l}^{l+l_{e}} \frac{\bar{C}_{k e}(l)}{\bar{C}_{k e}(z)} d z+\varepsilon e^{s t} \Lambda_{23}(z, s)\right]
\end{aligned}
$$

and

$$
\begin{gathered}
\overline{\Delta p}_{34 g}=g_{e} \bar{\rho}_{m e}(l) \int_{l}^{l+l e} \frac{\bar{C}_{k e}(l)}{\bar{C}_{k e}(z)} d z \\
\delta \Delta p_{34 g}=\varepsilon e^{s t} \Lambda_{23}(z, s)
\end{gathered}
$$

and

$$
\begin{gathered}
C_{r e}^{*}=\frac{\bar{\rho}_{m e}(l)}{\bar{\rho}_{m e}\left(l+l_{e}\right)}=\frac{\bar{C}_{k e}\left(l+l_{e}\right)}{\bar{C}_{k e}(l)} \\
\bar{\tau}_{34}=E_{e}\left(l+l_{e}\right)-E_{e}(l)=\frac{1}{\Omega_{e}} \ln C_{r e}^{*}
\end{gathered}
$$

The dimensionless form of Eq. (69) was given as

$$
\frac{\delta \Delta p_{34 g}^{*}}{\delta v^{*} v_{f i}^{*}}=\frac{1}{C_{r}^{*}} \frac{\Omega_{e}^{*}}{s^{*}-\Omega_{e}^{*}} \Lambda_{20}^{*}\left\{\frac{1}{N_{F r}} \frac{1}{\left[\left(\frac{1}{A_{e}^{*}}\right) C_{r}^{*}+V_{g j e}^{*} C_{r}^{*}+\frac{\Omega_{e}^{*} l_{e}^{*}}{v_{f i}^{*}}\right]}+\left[\frac{g^{*}}{s^{*} v_{f i}^{*}}\left(1-e^{-s^{*-\tau^{*}}+34}\right)\right]\right\}
$$

and

$$
\Lambda_{20}^{*}=\frac{1}{A_{e}^{*}} \Lambda_{5}^{*}-C_{r}^{* 2} \frac{V_{g j e}^{*}}{1+V_{g j}^{*}}\left\{\frac{1}{s^{*}-1} \frac{1}{C_{r}^{* 2}} \Lambda_{3}^{*}-e^{-\left[\left(s^{*}+1\right)^{-*} \tau_{23}^{* s^{* *}} \tau_{12}\right]}\right\}
$$




$$
\begin{gathered}
\Lambda_{5}^{*}=\Lambda_{3}^{*}+\frac{1}{s^{*}-1} \frac{V_{g j}^{*}}{1+V_{g j}^{*}}\left\{\Lambda_{3}^{*}-e^{-\left[\left(s^{*}-1\right)^{\tau_{23}^{*}+s^{*} \tau_{12}^{*}}\right]}\right\} \\
\Lambda_{3}^{*}=1-\frac{\left(1-e^{-s^{*} \tau_{12}^{*}}\right)}{s^{*}}
\end{gathered}
$$

where the characteristic functions $\Lambda_{3}^{*}$ and $\Lambda_{5}^{*}$ were derived by Ishii [19]. Also some dimensionless parameters were listed in Appendix B.

\subsection{Dimensionless Characteristic Functions for the Whole System}

The perturbations for the pressure drops in Regions A, B, and C and inlet velocity had been derived by Ishii [19] from the field equations and non-dimensionalized as For the upstream un-heated Region A

$$
\frac{\delta \Delta p_{01}^{*}}{\delta v^{*} v_{f i}^{*}}=2 k_{i}+\left(\frac{l_{o}^{*}}{A_{o}^{*}}\right) \frac{1}{v_{f i}^{*}} s^{*}+\frac{f_{o}}{2 D_{o}^{*}} 2 l_{o}^{*}\left(\frac{1}{A_{o}^{*}}\right)^{2}
$$

For the liquid heated Region B

$$
\frac{\delta \Delta p_{12}^{*}}{\delta v^{*} v_{f i}^{*}}=\tau_{12} s^{*}+\frac{f_{s}}{2 D_{o}^{*}} 2 \lambda^{*}+\left(\frac{v_{f i}^{*}}{N_{F r}}+\frac{f_{s}}{2 D_{o}^{*}} v_{f i}^{*}\right)\left(1-\Lambda_{3}^{*}\right)
$$

For the heated mixture Region C

$$
\begin{aligned}
& \frac{\delta \Delta p_{23}^{*}}{\delta v^{*} v_{f i}^{*}}=\left\{\ln C_{r}^{*}\left[\left(1+V_{g j}^{*}\right)+V_{g j}^{*}\left(\frac{1}{s^{*}-1}\right)\right] s^{*} \Lambda_{3}^{*}-V_{g j}^{*}\left(\frac{1}{s^{*}-1}\right)^{2} s^{*} C_{2}^{*}\right\} \\
& +\left\{-\frac{1}{1+V_{g j}^{*}}\left(1-\Lambda_{3}^{*}\right)+\ln C_{r}^{*}\left(\frac{s^{*}}{s^{*}-1}\right) \Lambda_{3}^{*}-\left[\left(\frac{1}{s^{*}-1}\right)^{2}-\frac{V_{g j}^{*}}{1+V_{g j}^{*}}\left(\frac{1}{s^{*}-1}\right)\right] C_{2}^{*}\right\} \\
& +\left(\frac{v_{f i}^{*}}{N_{F r}}\right)\left\{\left(1-\frac{1}{C_{r}^{*}}\right) \frac{\Lambda_{3}^{*}}{s^{*}-1}-\frac{1}{s^{*}}\left(\frac{1}{s^{*}-1}\right) C_{1}^{*}-\left(1-\Lambda_{3}^{*}\right)\right\} \\
& +\frac{\bar{f}_{m}}{2 D^{*}}\left\{\left(1-\lambda^{*}\right)\left(2 s^{*}-\frac{1}{1+V_{g j}^{*}}\right) \frac{\Lambda_{3}^{*}}{s^{*}-1}-v_{f i}^{*}\left(1+2 V_{g j}^{*}\right)\left(\frac{1}{s^{*}-1}\right)\left(\frac{1}{s^{*}-2}\right) C_{3}^{*}\right\} \\
& -\frac{f_{s}}{2 D^{*}} v_{f i}^{*}\left(1-\Lambda_{3}^{*}\right)-\rho_{g}^{*} \frac{V_{g j}^{* 2}}{1+V_{g j}^{*}}\left(2 C_{r}^{*}-1\right) \frac{C_{4}^{*}}{s^{*}-1}
\end{aligned}
$$


Then the total pressure drop perturbations can be expressed as

$$
\delta \Delta p_{e x}^{*}=\delta \Delta p_{01}^{*}+\delta \Delta p_{12}^{*}+\delta \Delta p_{23}^{*}+\delta \Delta p_{34}^{*}
$$

\section{EXPERIEMTNAL STABILITY MAPS}

The experimental stability boundary had been obtained from previous quasi-steady state tests using the natural circulation test facility as shown in Fig. 5. This test facility was designed and constructed to perform both transient and quasi-steady flow instability tests for the NMR. The detailed scaling analysis and design could be referred to our previous work [12, 15]. For the quasisteady tests, the test power and inlet flow conditions were important boundary conditions. The maximum power provided by four electric heaters $(2 \times 2)$ is $20 \mathrm{~kW}$, which was powered with a three-phase 480VAC source and controlled by Watlow SCR power controller with an accuracy of $\pm 0.5 \%$ of its actual power output. A similar tube-in-tube exchanger installed on the top of the downcomer worked as a cooler to reduce the temperature of the separated flow. Another threephase preheater of $18 \mathrm{~kW}$ was installed in the downcomer to adjust the inlet flow temperatures. The designed system pressure was $1 \mathrm{MPa}$ and can be controlled by the condenser, which was connected to the steam dome on the top and the downcomer at the bottom. Besides, this test facility was equipped with instruments to obtain various thermal hydraulic parameters, such as local temperatures, absolute pressures, pressure differentials, local void fractions, and mass flow rates [12].

The quasi-steady tests had been performed under various conditions by changing system pressure, core inlet subcooling, core inlet flow resistance coefficient, and core heat power etc. Based on certain stability criteria, the flow stability for a certain operating condition could be determined from the time trace profile of the loop natural circulation flow rate, which was defined as the

single-phase core inlet flow velocity. All those stable or unstable experimental conditions were plotted in the dimensionless stability plane, which also forms an experimental stability map. 



Fig. 5. Schematic of the Steady State Facility

A typical experimental stability map at a pressure of $200 \mathrm{kPa}$ can be seen in Fig. 6. A group of experimental conditions connected by a dash line stands for a fixed core heat flux during the quasisteady state tests. At a fixed core heat flux, the flow conditions went through the single-phase natural circulation in high-subcooling region, flashing instability, and two-phase natural circulation in low-subcooling region. Due to the significant increase of the natural circulation rate in the two-phase flow, the phase change number were largely reduced in this region. In addition, the stable conditions are marked with the blue color, while the unstable ones are marked with the red color in Fig. 6. The intermittent flow oscillations were mainly caused by the flashing instability occurring during the transfer between the stable single-phase natural circulation and stable twophase natural circulation. As can be seen, the stability boundary in this case were very close to the zero-quality line at the riser or chimney outlet. Since the zero-quality line at the core exit was derived based on the assumption of the thermal and mechanical equilibrium conditions, the shift of stability boundary could be caused by the non-equilibrium phenomenon of subcooled boiling in 
the core section. Similarly, Figure 7 shows the experimental stability map at $400 \mathrm{kPa}$. The general trends were very similar to the stability map at $200 \mathrm{kPa}$. However, the stability boundary moved to the zero-quality line at the core exit. The flashing effect was highly suppressed at a higher pressure due to reduced thermal non-equilibrium. Moreover, the whole unstable region of the transition phase was very thin in the experimental stability plane at the pressure of $400 \mathrm{kPa}$.

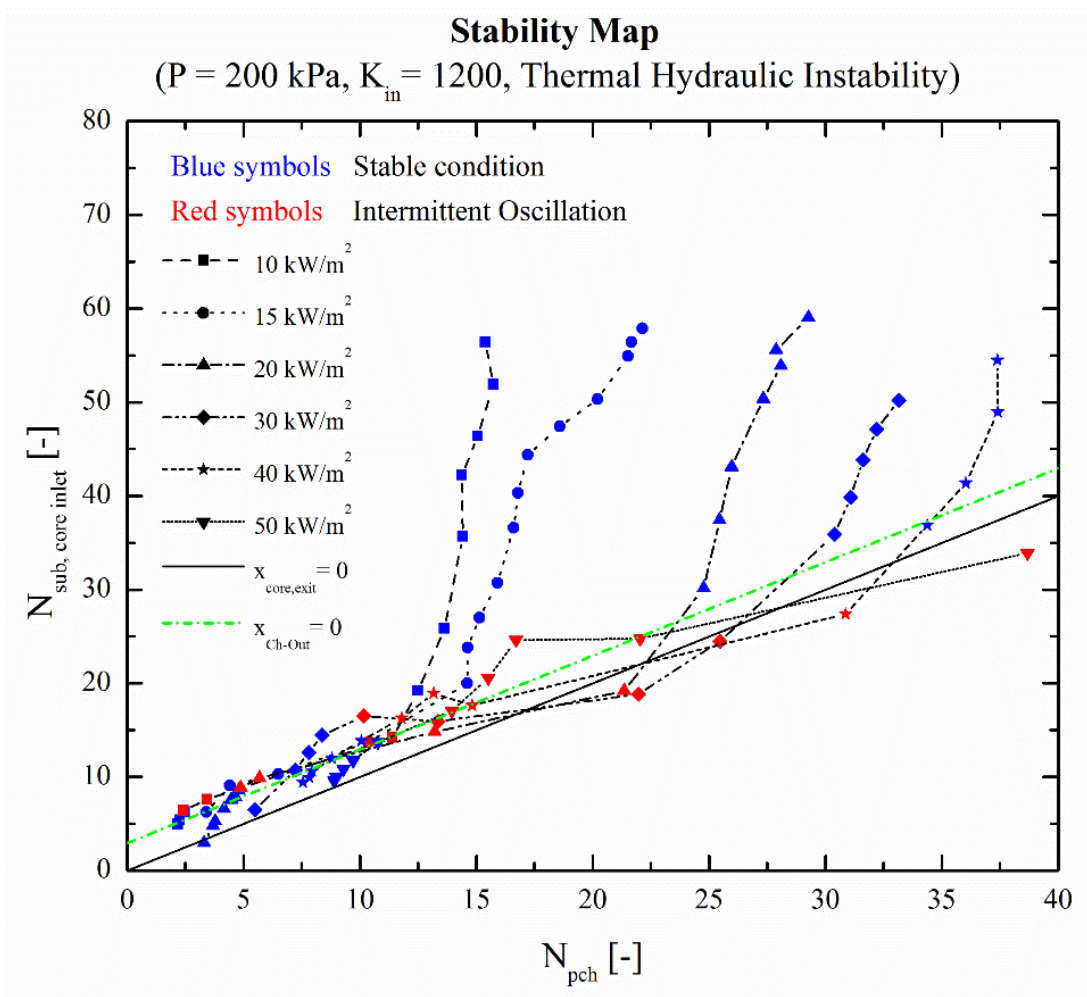

Fig. 6. Experimental stability at $200 \mathrm{kPa}$ 


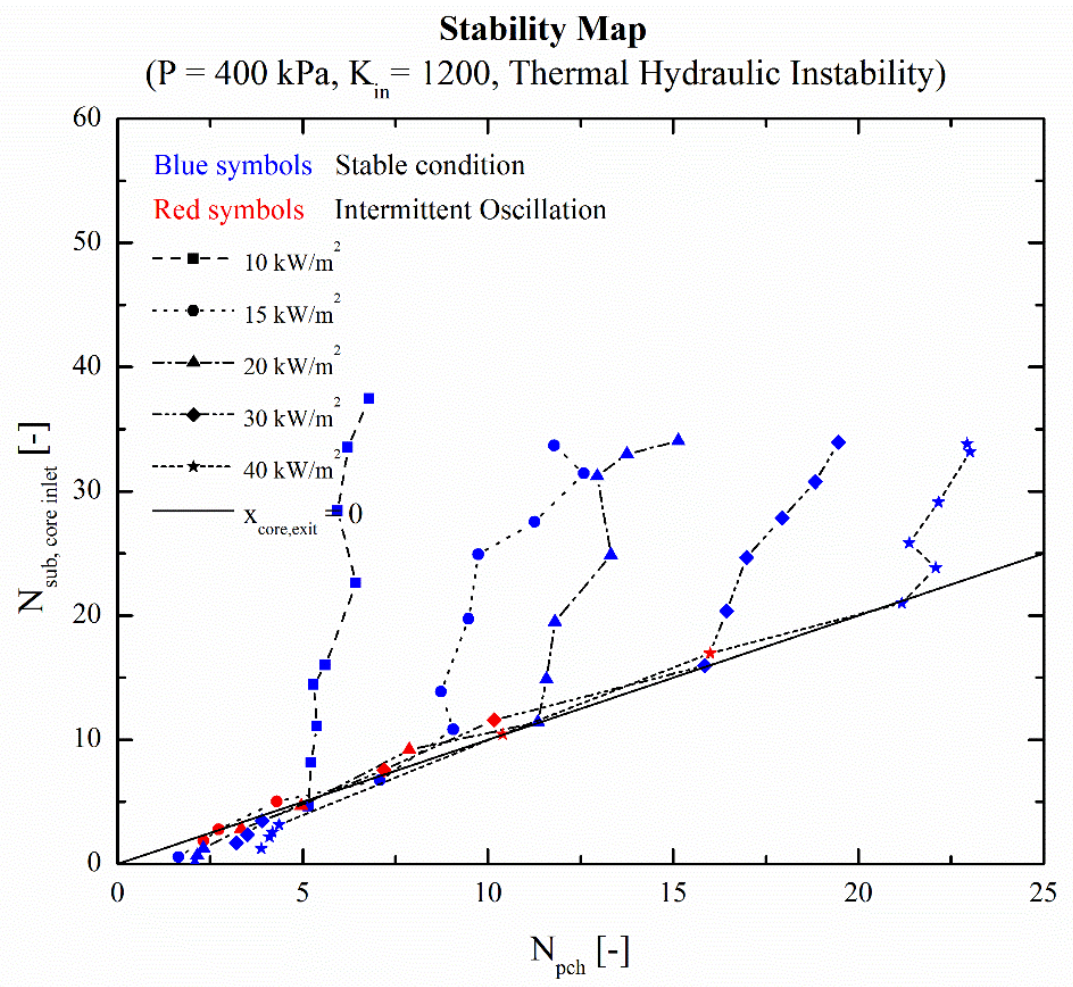

Fig. 7. Experimental stability at $400 \mathrm{kPa}$

\section{APPLICATION OF FREQUENCY DOMAIN ANALYSIS}

To verify and validate the flashing model, the frequency domain analysis was implemented to the natural circulation test facility designed to investigate the flow instability for the NMR under the low power and low pressure conditions. The geometric parameters and operation conditions of the test facility were used as the system input parameters of the numerical stability analysis. The inlet flow conditions and system power were determined by the subcooling number and phase change number, respectively. By applying the linear stability analysis, the theoretical stability boundary could be determined numerically by using the D-partition method on the dimensionless characteristic equation for the perturbations between the total pressure drop and inlet velocity. In addition, the parametric stability analysis were performed on the effects of system pressure and inlet flow resistance etc. on the flow instability. It should be noted that the drift velocity was neglected in current analysis since flashing occurs at very low void fraction conditions, which was almost single-phase natural circulation observed from our previous measurements [13, 14]. 
Figure 8 shows the numerical stability map at two different core inlet flow resistance coefficients, i.e., $K_{\text {in }}=1200$ and 600 , at a system pressure of $200 \mathrm{kPa}$. As can be seen, the stability boundaries are plotted in the dimensionless plane of $\mathrm{N}_{\text {sub }}-\mathrm{N}_{\mathrm{pch}}$. In addition, the zero-quality line at the core exit, where two dimensionless numbers are equal, is also included in the stability plane. In the case of $K_{\text {in }}=600$, two boundaries could be obtained, including a flashing boundary and a density wave oscillations (DWO) boundary, in current ranges of the plane. The flashing boundary was located closely below the zero-quality line at the core exit, while the DWO boundary moved to the right of the stability plane with higher phase change number. In the case of $K_{i n}=1200$, only a flashing boundary could be obtained in current range of the stability plane. The DWO boundary for $K_{\text {in }}=$ 1200 moved to the right part with higher phase change number, which exceed the ranges of current dimensionless stability plane. In other words, the stable region was expanded by increasing the inlet flow resistance coefficient, which was also verified by Ishii [19].

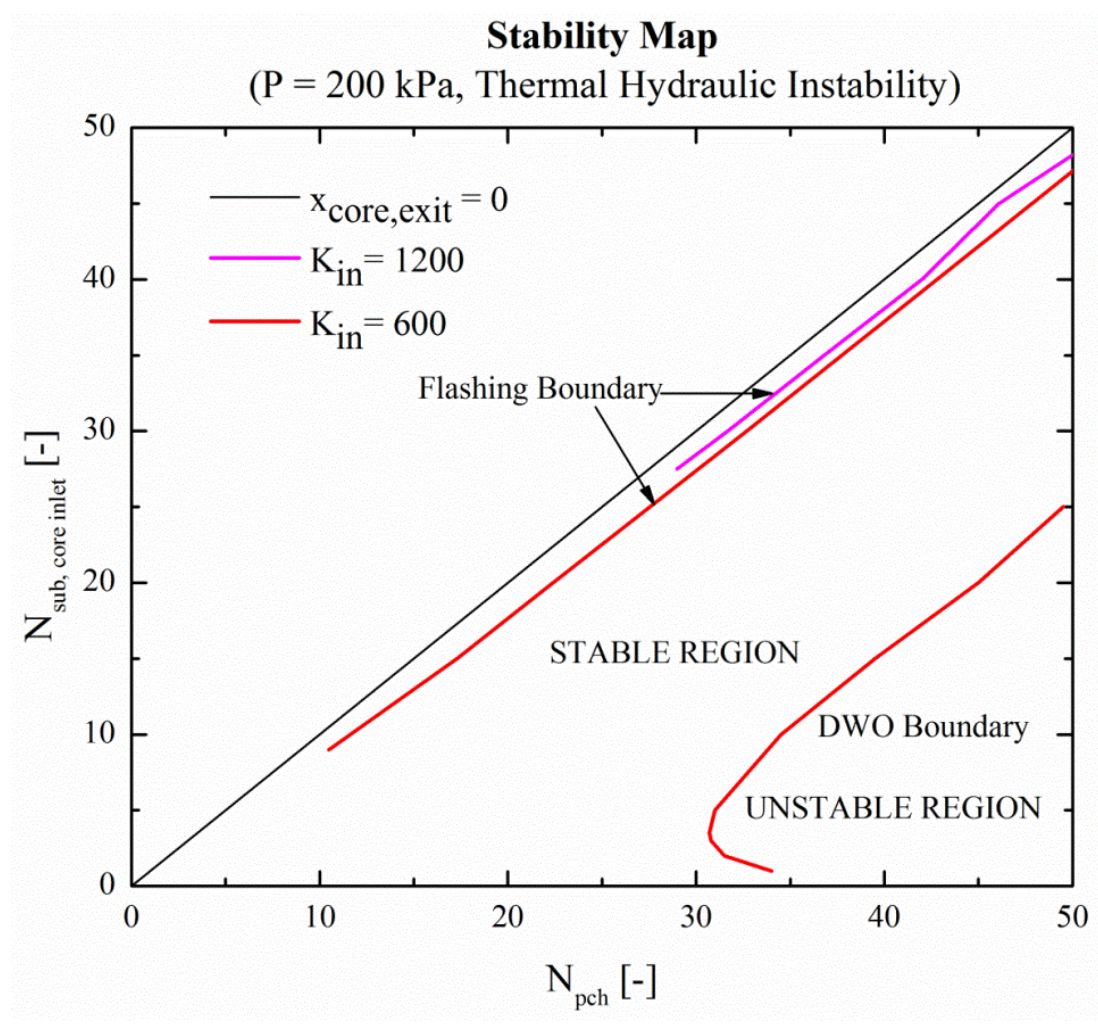

Fig. 8. Stability Map at $200 \mathrm{kPa}$ 
The pressure effects on the theoretical stability boundary was also carried out in the frequency domain analysis. Figure 9 shows the stability boundary at the system pressure of $400 \mathrm{kPa}$ with a flow resistance of $K_{\text {in }}=600$. As can be seen in Fig. 9, only the DWO boundary could be numerically predicted in the dimensionless stability plane. Moreover, the pressure effect on the DWO boundary was not significant, which has also been confirmed by other researchers [19]. By comparing with the experimental stability boundary in Fig. 7, the flashing boundary at high pressure still existed and could be presented by the zero-quality line at the core exit by comparing with experimental data at $400 \mathrm{kPa}$.

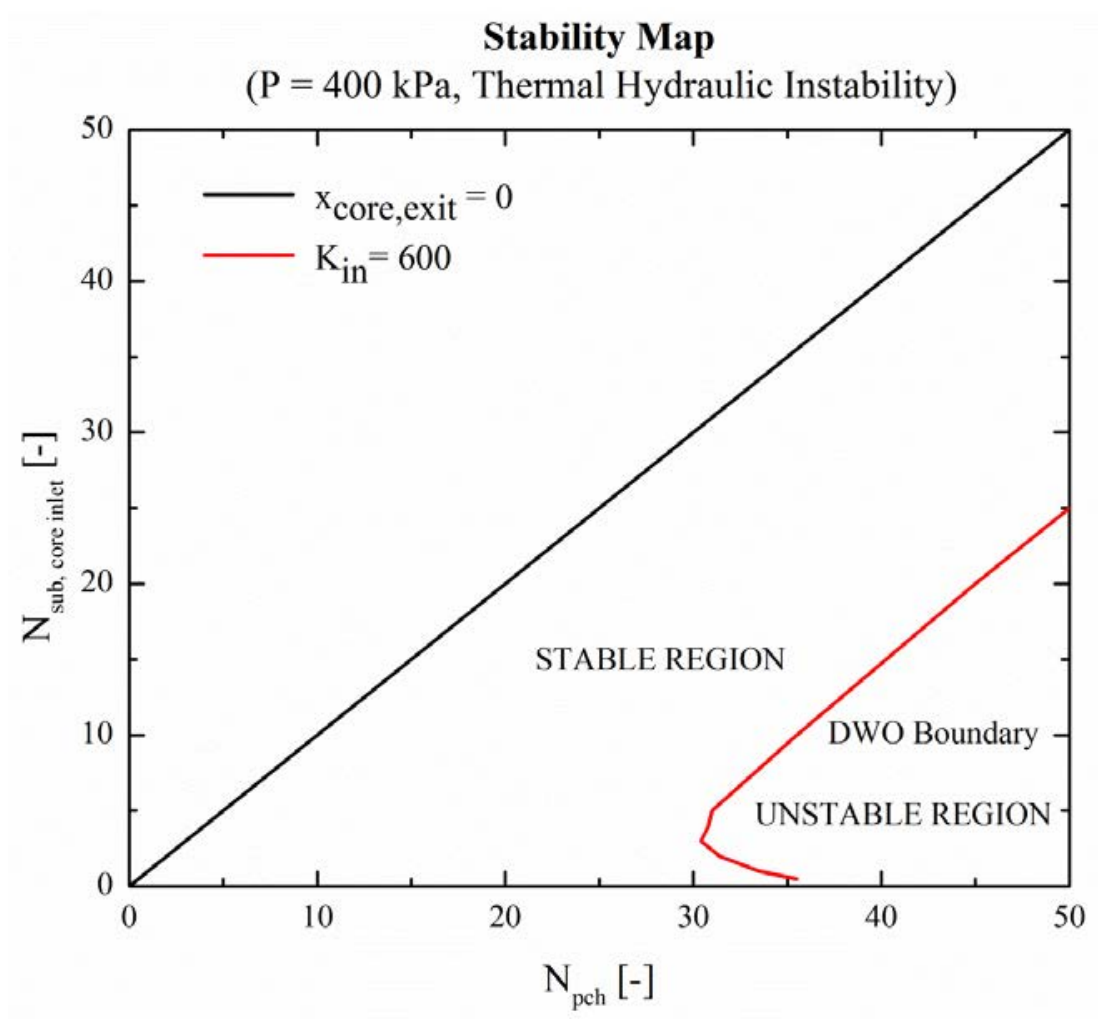

Fig. 9. Stability Map at $400 \mathrm{kPa}$

Although the theoretical stability boundaries including the DWO boundary and flashing boundary can be obtained from the frequency domain analysis. Uncertainties of the flashing boundary still exists due to the complexities of the flow instability itself. The flashing boundary predicted in this study is similar to the Type-I flow instability observed from other researchers [22-24]. In current 
study, the theoretical flashing stability boundary in Fig. 8 does not agree very well with the one in Fig. 6 from the quasi-steady state tests, where the flashing boundary is above the zero quality line at the core exit. One possible reason is that the thermal non-equilibrium conditions, i.e., subcooled boiling, is not yet considered in current frequency domain analysis. Thus the non-boiling length $\bar{\lambda}$ can be overestimated in current frequency domain analysis considering thermal equilibrium conditions. Some preliminary analysis has been done to justify this claim by manually reducing the non-boiling length in the frequency domain analysis code. However, a more detailed analysis by taking account of the thermal non-equilibrium will be performed in the future.

\section{CONCLUSIONS}

Flashing instability is normally caused by the reduced hydrostatic head along the riser or chimney in the natural circulation system. In order to predict the flashing stability, the frequency domain analysis was performed to obtain the stability boundary in the dimensionless stability plane with the newly developed flashing model. Following Ishii's approach to obtain the density wave oscillations (DWO) boundary, the coolant enthalpy change rate during the flashing was considered as an axially uniform heat source based on the water P-T diagram. The kinematic and dynamics of the downstream unheated mixture region (riser or chimney) were obtained. In addition, the pressure drop response was given in dimensionless equations for the stability analysis. The system transfer functions were obtained for the pressure perturbations with the inlet flow velocity perturbations. The stability boundary were obtained by determining the nature of roots of the system characteristic equations with D-partition method.

In the dimensionless stability plane of $\mathrm{N}_{\text {sub }}-\mathrm{N}_{\mathrm{pch}}$, two kinds of stability boundaries, i.e., the flashing instability boundary and the density wave oscillations boundary, were predicted using frequency domain analysis for the natural circulation test facility. Among them, DWO boundary has been widely studied and confirmed in current analysis. The theoretical flashing instability boundary was closely parallel to the zero-quality line at the core exit at the pressure of $200 \mathrm{kPa}$. However, the theoretical flashing boundary was not able to be numerically predicted at the pressure of $400 \mathrm{kPa}$, which was considered to match with the zero-quality line under high pressure. In addition, the predicted stability boundaries were compared with the quasi-steady state experimental data 
acquired from the natural circulation test facility. The biggest difference was that the experimental flashing boundary was located above the zero-quality line at the core exit, while the theoretical boundary was parallel below the line. The possible reason was that the thermal equilibrium was assumed in current study, whereas the subcooled boiling in the core section could occur in real quasi-steady state tests. Although the theoretical flashing boundary showed some discrepancy with the experimental data, the effect of system pressure and inlet $\mathrm{K}$ factor could be correctly simulated by using current frequency domain analysis for the natural circulation test facility. In the future, the frequency domain analysis including both the flashing model and thermal non-equilibrium will be carried out and validated against existing instability experimental data.

\section{Nomenclature}

\section{$\underline{\text { Latin Letters }}$}

$\begin{array}{ll}A & \text { flow area }\left[\mathrm{m}^{2}\right] \\ C & \text { specific heat [J/kg-K] } \\ C_{k} & \text { kinematic wave velocity [m/s] } \\ C_{p} & \text { specific heat at constant pressure [J/kg-K] } \\ D & \text { hydraulic diameter [m] } \\ f & \text { friction factor or frequency [-] } \\ g & \text { gravitational acceleration }\left[\mathrm{m} / \mathrm{s}^{2}\right] \\ i_{f g} & \text { latent heat of vaporization }[\mathrm{J} / \mathrm{kg}] \\ j & \text { volumetric flux or center-of-volume velocity [m/s] } \\ K & \text { K factor (Minor loss coefficient) [-] } \\ l & \text { heated section length [m] } \\ L & \text { axial length scale [-] } \\ \dot{\mathrm{m}} & \text { mass flow rate [kg/s] } \\ \mathrm{N} & \text { dimensionless number [-] } \\ \mathrm{N}_{\mathrm{Fr}} & \text { Froude number [-] } \\ \mathrm{N}_{\mathrm{fl}} & \text { flashing number [-] }\end{array}$




\begin{tabular}{|c|c|}
\hline $\mathrm{N}_{\text {sub }}$ & subcooling number [-] \\
\hline $\mathrm{N}_{\mathrm{Zu}}$ & Zuber (phase change) number [-] \\
\hline$p$ & pressure $[\mathrm{Pa}]$ \\
\hline$q$ & power $[\mathrm{w}]$ \\
\hline$q^{\prime \prime}$ & heat flux $\left[\mathrm{w} / \mathrm{m}^{2}\right]$ \\
\hline$q^{\prime \prime \prime}$ & volumetric heat generation rate $\left[\mathrm{w} / \mathrm{m}^{3}\right]$ \\
\hline$s$ & complex number $[\mathrm{a}+\mathrm{bi}]$ \\
\hline$t$ & time $[\mathrm{s}]$ \\
\hline$T$ & temperature $\left[\mathrm{K}\right.$ or $\left.{ }^{\circ} \mathrm{C}\right]$ \\
\hline$v$ & velocity $[\mathrm{m} / \mathrm{s}]$ \\
\hline$V$ & volume $\left[\mathrm{m}^{3}\right]$ \\
\hline$V_{g j}$ & drift velocity $[\mathrm{m} / \mathrm{s}]$ \\
\hline$z$ & axial coordinate $[\mathrm{m}]$ \\
\hline
\end{tabular}

\section{Greek}

$\alpha$

$\Gamma_{g}$

$\Delta$

$\varepsilon$

$\bar{\lambda}$

$\Lambda_{n}$

$\mu$

$\nu$

$\xi$

$\rho \quad$ density $\left[\mathrm{m}^{3} / \mathrm{s}\right]$

$\tau \quad$ time scale [-]

$\tau_{01,12,23,34,13}$ residence time in Regions (A), (B), (C), (D), and in the heated section [s]

void fraction [-]

mass generation for the vapor phase $\left[\mathrm{kg} / \mathrm{m}^{3}-\mathrm{s}\right]$

difference [-]

infinitesimal [-]

non-boiling length [m]

various transfer function [-]

dynamic viscosity $[\mathrm{kg} / \mathrm{m}-\mathrm{s}]$

kinematic viscosity $\left[\mathrm{m}^{2} / \mathrm{s}\right]$

perimeter $[\mathrm{m}]$ 


$\begin{array}{ll}\Omega & \text { characteristic frequency [-] } \\ \omega & \text { frequency [-] }\end{array}$

$\underline{\text { Superscripts }}$

* dimensionless

\section{$\underline{\text { Subscripts }}$}

\begin{tabular}{|c|c|}
\hline$e$ & exit/Region D \\
\hline$f$ & liquid \\
\hline$f l$ & flashing \\
\hline$g$ & gas \\
\hline$h$ & heated \\
\hline$i$, in & inlet \\
\hline$k$ & each phase \\
\hline$m$ & mixture \\
\hline me & mixture in Region D \\
\hline$o$ & reference point/component \\
\hline$S$ & saturation \\
\hline$s u b$ & subcooling \\
\hline$W$ & Wall \\
\hline$w$ & wetted \\
\hline 3 & bottom of Region D \\
\hline 4 & top of Region D \\
\hline 12 & Region B \\
\hline 23 & Region C \\
\hline 34 & Region D \\
\hline
\end{tabular}

\section{Operators}


time average

$\frac{D_{k}}{D_{t}} \quad=\frac{\partial}{\partial t}+v_{k} \cdot \nabla$ 


\section{Acknowledgements}

This material is based upon work supported under a Department of Energy Nuclear Energy University Program.

\section{References}

[1] S. Shi, T. Hibiki, and M. Ishii, "Startup instability in natural circulation driven nuclear reactors,” Progress in Nuclear Energy, 90, pp. 140-150, Jul. 2016.

[2] D. D. Lisowski, R. M. Scherrer, T. C. Haskin, M. H. Anderson, M. L. Corradini, S. M. Albiston, and A. Tokuhiro, "Experimental studies of NGNP reactor cavity cooling system with water,” in Proceedings of ICAPP 2011, France, pp. 2851.

[3] F. Inada, M. Furuya, and A. Yasuo, "Thermo-hydraulic instability of boiling natural circulation loop induced by flashing (analytical consideration)," Nuclear Engineering and Design, 200, no. 1-2, pp. 187-199, Aug. 2000.

[4] M. Furuya, F. Inada, and T. H. J. J. van der Hagen, "Flashing-induced density wave oscillations in a natural circulation BWR-mechanism of instability and stability map," Nuclear Engineering and Design, 235, no. 15, pp. 1557-1569, Jul. 2005.

[5] A. Manera, U. Rohde, H.-M. Prasser, and T. H. J. J. van der Hagen, "Modeling of flashinginduced instabilities in the start-up phase of natural-circulation BWRs using the two-phase flow code FLOCAL,” Nuclear Engineering and Design, 235, no. 14, pp. 1517-1535, Jun. 2005.

[6] R. Hu and M. S. Kazimi, "Flashing-induced instability analysis and the start-up of natural circulation boiling water reactors,” Nuclear Technology, 176, no. 1, pp. 57-71, Oct. 2011.

[7] J. D. Lee, C. Pan, and S. W. Chen, "Nonlinear dynamic analysis of a two-phase natural circulation loop with multiple nuclear-coupled boiling channels," Annals of Nuclear Energy, 80, pp. 77-94, Jun. 2015.

[8] G. Su, D. Jia, F. Kenji, and Y. Guo, "Theoretical and experimental study on density wave oscillation of two-phase natural circulation of low equilibrium quality," Nuclear Engineering and Design, 215, no. 3, pp. 187-198, Jun. 2002.

[9] X. Guo, Z. Sun, J. Wang, and S. Yu, "Steady-state performances and scaling analyses for an open flashing-driven natural circulation system," Progress in Nuclear Energy, 87, pp. 1-14, 2016. 
[10] M. Ishii, S. Shi, W. S. Yang, Z. Wu, S. Rassame, and Y. Liu, "Novel modular natural circulation BWR design and safety evaluation," Annals of Nuclear Energy, 85, pp. 220-227, Nov. 2015.

[11] Z. Wu, W. Si. Yang, S. Shi, and M. Ishii, “A core design study for a small modular boiling water reactor with long-life core,” Nuclear Technology, 193, no. 3, Mar. 2016.

[12] F. Y. Odeh and W. S. Yang, "Core design optimization and analysis of the Purdue Novel Modular Reactor (NMR-50),” Annals of Nuclear Energy, 94, pp. 288-299, Aug. 2016.

[13] S. Shi, J. P. Schlegel, C. S. Brooks, Y.-C. Lin, J. Eoh, Z. Liu, Q. Zhu, Y. Liu, T. Hibiki, and M. Ishii, "Experimental investigation of natural circulation instability in a BWR-type small modular reactor,” Progress in Nuclear Energy, 85, pp. 96-107, Nov. 2015.

[14] S. Shi, Z. Wu, Z. Liu, J. P. Schlegel, C. S. Brooks, J. Eoh, Y. Yan, Y. Liu, W. S. Yang, and M. Ishii, "Experimental investigation of natural circulation instability with void-reactivity feedback during startup transients for a BWR-type SMR,” Progress in Nuclear Energy, 83, pp. 73-81, Aug. 2015.

[15] S. Shi, C. Brooks, J. Eoh, and M. Ishii, "Pressurized startup transient analyses for the BWRtype NMR-50,” Transactions- American Nuclear Society, 111, pp. 1613-1615.

[16] S. Shi, Y.-C. Lin, W. S. Yang, and M. Ishii, "Experimental stability maps for a BWR-type small modular reactor," in the 16th International Topical Meeting on Nuclear Reactor Thermal Hydraulics (NURETH-16), Chicago, IL, 2015.

[17] S. Shi, "Investigation of natural circulation instability and transients in passively safe Novel Modular Reactor,” Dissertation, Purdue University, West Lafayette, IN, 2015.

[18] N. Zuber, "Flow excursions and oscillations in boiling, two-phase flow systems with heat addition,” in Symposium on Two-phase Flow Dynamics, pp. 1071-1089, 1967.

[19] M. Ishii, "Thermally induced flow instabilities in two-phase mixtures in thermal equilibrium,” Dissertation, Georgia Institute of Technology, 1971.

[20] P. Saha, "Thermally induced two-phase flow instabilities, including the effect of thermal non-equilibrium between the phases,” Dissertation, Georgia Institute of Technology, 1974.

[21] J.L. Achard, D.A. Drew, and R.T. Lahey, "The effect of gravity and friction on the stability in a channel,” Chemical Engineering Communications, 11 (1-3), pp. 59-79.

[22] D. D. B. van Bragt and T. H. J. J. van der Hagen, "Stability of natural circulation boiling water reactors: Part I - description stability model and theoretical analysis in terms of dimensionless groups,” Nuclear Technology, 121, no. 1, pp. 40-51, Jan. 1998.

[23] T. H. J. J. van der Hagen, D. D. B. van Bragt, F. J. van der Kaa, J. Karuza, D. Killian, W. H. M. Nissen, A. J. C. Stekelenburg, and J. A. A. Wouters, "Exploring the Dodewaard type-I and type-II stability; from start-up to shut-down, from stable to unstable,” Annals of Nuclear Energy, 24, no. 8, pp. 659-669, May 1997. 
[24] F.-S. Wang, L.-W. Hu, and C. Pan, "Thermal and stability analysis of a two-Phase natural circulation loop,” Nuclear Science and Engineering, 117, no. 1, pp. 33-46, May 1994. 